\title{
REFEIÇÕES SERVIDAS EM UNIDADE DE ALIMENTAÇÃO E NUTRIÇÃO: UMA AVALIAÇÃO DA SAÚDE DOS TRABALHADORES
}

\author{
Meals served at a food and nutrition service: an evaluation of \\ workers' health
}

\author{
Comidas de la unidad de alimentación y nutrición: una \\ evaluación de la salud de los trabajadores
}

\begin{abstract}
RESUMO
Objetivo: Avaliar a composição nutricional das refeições servidas em uma Unidade de Alimentação e Nutrição em relação aos parâmetros nutricionais estabelecidos pelo Programa de Alimentação do Trabalhador e avaliar os fatores de risco associados às Doenças Crônicas Não Transmissíveis existentes nos beneficiados. Métodos: Realizou-se estudo observacional, transversal e descritivo em uma Unidade de Alimentação e Nutrição, localizada em uma empresa do setor de prestação de serviços automotivos, no município de Curitiba-PR, em outubro de 2014. Para a coleta de dados, obtiveram-se informações referentes à composição nutricional das refeições servidas no período do almoço, durante um mês. Realizou-se avaliação antropométrica e aplicou-se um questionário para avaliar o estilo de vida de 19 funcionários da empresa. Resultados: Na média geral, o valor energético total $(1.311,7$ Kcal), proteínas $(19 \%)$, gorduras totais $(32 \%)$, fibras $(21,14 \mathrm{~g})$, sódio $(1828,6 \mathrm{mg})$ e o percentual proteico-calórico (12\%) das refeições estavam acima dos limites estabelecidos pelo Programa de Alimentação do Trabalhador. Constatou-se que 53\% dos funcionários estavam com sobrepeso, $21 \%$ eram fumantes, $58 \%$ não praticavam atividade física e $32 \%$ apresentavam alguma patologia, sendo o diagnóstico de hipertensão arterial relatado por todos os funcionários. Conclusão: As refeições servidas na Unidade de Alimentação e Nutrição avaliada estavam inadequadas aos parâmetros estabelecidos para a alimentação do trabalhador, o que pode acarretar prejuízo à saúde dos beneficiários quando associado aos principais fatores de riscos encontrados, como sobrepeso, sedentarismo e prevalência de hipertensão arterial, além do não cumprimento à legislação trabalhista brasileira.
\end{abstract}

Descritores: Programas e Políticas de Nutrição e Alimentação; Doença Crônica; Alimentação Coletiva; Estado Nutricional.

\section{ABSTRACT}

Objective: To evaluate the nutritional composition of meals served at a Food and Nutrition Service in relation to the nutritional parameters established by the Worker's Food Program, and to evaluate the risk factors associated to the non-communicable chronic diseases found among the beneficiaries of the service. Methods: An observational, cross-sectional and descriptive study conducted at the Food and Nutrition Service, located at a company that provides automotive services in the municipality of Curitiba, Paraná (PR), in October 2014. For data collection, information regarding the nutritional composition of meals served at midday was gathered. An anthropometric evaluation was conducted, and a questionnaire was applied to evaluate the lifestyle of 19 company employees. Results: In general, the total energy value $(1,311.7 \mathrm{Kcal})$, proteins $(19 \%)$, total fats $(32 \%)$, fibers $(21.14 \mathrm{~g})$, sodium $(1,828.6 \mathrm{mg})$, and the percentage of calories from proteins $(12 \%)$ in the meals served were above the limits established by the Worker's-Food Program. It was found that 53\% of the employees were overweight, $21 \%$ were smokers, $58 \%$ did not practice physical activity, and $32 \%$ presented some pathological condition, with the diagnosis of arterial hypertension reported by all the employees. Conclusion: The meals served at the Food and Nutrition Service evaluated did not meet the parameters established for worker's nutrition, which could
Artigo Original

Priscila de Lima Batista ${ }^{(1)}$ Lize Stangarlin ${ }^{(2)}$

Laissa Benites Medeiros ${ }^{(3)}$

Ana Lúcia Serafim( ${ }^{(4)}$

Naína Lopes de Souza de Jesus ${ }^{(5)}$

Caroline dos Santos Peixoto ${ }^{(6)}$ Mariane Rossato Moreira ${ }^{(7)}$

1) Centro Universitário Campos de Andrade - Uniandrade - Curitiba (PR) - Brasil

2) Universidade Federal do Paraná - UFPR Curitiba (PR) - Brasil

3) Universidade de Caxias do Sul - UCS Caxias do Sul (RS) - Brasil

4) Universidade Federal de Santa Maria UFSM - Santa Maria (RS) - Brasil

5) Universidade Tuiuti do Paraná - UTP Curitiba (PR) - Brasil

6) Centro Universitário Franciscano - Santa Maria (RS) - Brasil

7) Universidade Federal do Rio de Janeiro UFRJ - Rio de Janeiro (RJ) - Brasil

Recebido em: 30/05/2015 Revisado em: 12/08/2015 Aceito em: 21/11/2015 
harm the health of beneficiaries, when associated to the main risk factors found among them, such as excess weight, sedentary lifestyle, and prevalence of arterial hypertension, besides the lack of compliance with Brazilian labor laws.

Descriptors: Nutrition Programs and Policies; Chronic Disease; Collective Feeding; Nutritional Status.

\section{RESUMEN}

Objetivo: Evaluar la composición nutricional de las comidas de la Unidad de Alimentación y Nutrición respecto los parámetros de nutrición establecidos en el Programa de Alimentación del Trabajador y evaluar los factores de riesgo asociados a las Enfermedades Crónicas No Trasmisibles de los beneficiados. Métodos: Se realizó un estudio observacional, transversal y descriptivo en una Unidad de Alimentación y Nutrición localizada en una empresa del sector de servicio automotriz, en el municipio de Curitiba-PR en octubre de 2014. Para la recogida de datos se obtuvieron informaciones respecto la composición nutricional de las comidas servidas en el almuerzo durante un mes. Se realizó la evaluación antropométrica y se aplicó un cuestionario para evaluar el estilo de vida de 19 empleados de la empresa. Resultados: En la media general, el valor energético total (1.311,7 Kcal), proteinas (19\%), las grasas totales (32\%), las fibras $(21,14 \mathrm{~g})$, el sodio $(1828,6 \mathrm{mg}$ ) y el porcentual proteicocalórico (12\%) de las comidas estaban por encima de los límites establecidos en el Programa de Alimentación del Trabajador. Se constató que el 53\% de los trabajadores tenían sobrepeso, el 21\% eran fumadores, el 58\% no practicaban actividad fisica, el $32 \%$ presentaban alguna patología y el diagnostico de hipertensión arterial fue relatado por todos los trabajadores. Conclusión: Las comidas de la Unidad de Alimentación y Nutrición evaluada eran inadecuadas para los parámetros establecidos para la alimentación del trabajador lo que puede perjudicar a la salud de los beneficiarios al asociarlas a los principales factores de riesgo encontrados como el sobrepeso, el sedentarismo y la prevalencia de hipertensión arterial además del no cumplimiento de la legislación brasileña de trabajo.

Descriptores: Programas y Políticas de Nutrición y Alimentación; Enfermedad Crónica; Alimentación Colectiva; Estado Nutricional.

\section{INTRODUÇÃO}

A transição do perfil nutricional no Brasil é caracterizada pela alteração do padrão alimentar associado à redução da atividade física, o que reflete diretamente na mudança da composição corporal da população ${ }^{(1)}$. Associado a isso, verifica-se também aumento da prevalência de Doenças Crônicas Não Transmissíveis (DCNT), que constituem o problema de saúde de maior magnitude e correspondem a cerca de $70 \%$ das causas de mortes, atingindo fortemente camadas pobres da população e grupos mais vulneráveis, como a população de baixa escolaridade e renda ${ }^{(2)}$.
A Organização Mundial da Saúde (OMS) destaca que os principais fatores de risco para as DCNT são tabagismo, consumo excessivo de bebidas alcoólicas, dietas inadequadas, inatividade física e obesidade, as quais podem gerar morbidades como cardiopatias, diabetes mellitus, alguns tipos de câncer e doenças respiratórias crônicas ${ }^{(3)}$.

Com o intuito de mudar o cenário do país em relação às DCNT, o Ministério da Saúde (MS) iniciou em 2011 um plano de ações integrando vários setores para a prevenção e controle dessas doenças e seus fatores de risco ${ }^{(4)}$. Dentre as ações estão estratégias de apoio à implementação dos parâmetros nutricionais estabelecidos pelo Programa de Alimentação do Trabalhador (PAT), com foco na alimentação saudável e prevenção dessas patologias no ambiente de trabalho(5).

O PAT foi criado pelo governo federal por meio da Lei $\mathrm{n}^{\circ}$ 6.321, de 14 de abril de $1976^{(6)}$, e regulamentado pelo Decreto $\mathrm{n}^{\circ} 5$, de 14 de janeiro de $1991^{(7)}$. É considerado um programa de complementação alimentar, estruturado em parceria entre governo, empresas e trabalhadores, no qual se prioriza os de baixa renda, visando reduzir a incidência de acidentes de trabalho, diminuir a taxa de absenteísmo e rotatividade, bem como aumentar a produtividade nas empresas $^{(8)}$.

A adesão ao PAT é voluntária, mas para usufruir dos benefícios, as empresas beneficiárias devem elaborar suas refeições seguindo os parâmetros nutricionais exigidos pela Portaria Interministerial $n^{0} 66 / 2006^{(9)}$ e Portaria $n^{\circ}$ $193 / 2006^{(10)}$, as quais estabelecem critérios em relação à distribuição de macronutrientes, fibra e sódio nas refeições; quantitativo de frutas, legumes ou verduras; e a exigência de responsabilidade técnica (nutricionista) para a execução do programa.

O objetivo do estudo foi avaliar a composição nutricional das refeições servidas em uma Unidade de Alimentação e Nutrição em relação aos parâmetros nutricionais estabelecidos pelo PAT e avaliar os fatores de riscos associados às DCNT existentes nos beneficiados.

\section{MÉTODOS}

Estudo observacional, transversal, de caráter descritivo, realizado no período de outubro de 2014, em uma Unidade de Alimentação e Nutrição (UAN), localizada em uma empresa do setor de prestação de serviços automotivos, no município de Curitiba-PR, sendo selecionada por conveniência e critério de acesso.

A empresa apresenta modalidade de autogestão, com serviço tipo self-service, exceto para o prato proteico e sobremesa, que são porcionados, e fornece, em média, 400 refeições por dia, servidas no período do almoço. 
Utilizou-se como critério de inclusão o fato de a empresa ser beneficiária do PAT, dispor em período integral de nutricionista, ter interesse e disponibilidade em participar do estudo. Para a seleção dos funcionários, utilizou-se como critério idade superior a 18 anos, desempenhar atividades do tipo moderada na empresa, realizar as refeições na UAN e assinar o Termo de Consentimento Livre e Esclarecido (TCLE).

Para avaliação da composição nutricional das refeições, realizou-se levantamento das preparações servidas no horário do almoço, durante um mês (outubro/2014), com 20 dias úteis, divididos em quatro semanas, com atendimento de segunda a sexta-feira. Primeiramente, coletaramse informações sobre os ingredientes e a quantidade de alimentos in natura utilizados nas preparações; depois, avaliaram-se as medidas caseiras, fator de correção e cocção, por meio da Tabela de Medidas Caseiras do Instituto Brasileiro de Geografia e Estatística (IBGE) ${ }^{(11)}$, e calculouse o rendimento total das preparações ${ }^{(12)}$. O per capita foi definido a partir do quantitativo de alimentos produzidos dividido pelo número de refeições servidas ao $\operatorname{dia}^{(13)}$.

Posteriormente, calculou-se o Valor Energético Total (VET), as quantidades de carboidratos, proteínas, gorduras totais, gorduras saturadas, fibras e sódio ${ }^{(11,14)}$, e o percentual proteico-calórico (NdPCal) ${ }^{(15)}$ de cada refeição.

Os resultados obtidos foram comparados com os valores preconizados pela Portaria Interministerial $\mathrm{n}^{\circ} 66 / 2006^{(9)} \mathrm{e}$ Portaria $n^{\circ} 193 / 2006^{(10)}$, sendo apresentados no Quadro I.

Quadro I - Parâmetros nutricionais estabelecidos pelo Programa de Alimentação do Trabalhador para refeições principais (almoço, janta e ceia), 2015.

\begin{tabular}{|l|l|}
\hline Distribuição das variáveis nutricionais & \multicolumn{1}{|c|}{ Parâmetros estabelecidos } \\
\hline \multirow{3}{*}{ Valor Energético Total } & Deverão conter de 600 a 800 calorias, admitindo-se acréscimo de 20\% do \\
& $\begin{array}{l}\text { VET, de 2000 calorias/dia (400 calorias), e deverão corresponder à faixa de } \\
30 \% \text { a 40\% do Valor Energético Total diário }\end{array}$ \\
\hline Carboidratos & $60 \%$ do Valor Energético Total \\
\hline Proteínas & $15 \%$ do Valor Energético Total \\
\hline Gorduras totais & $25 \%$ do Valor Energético Total \\
\hline Gorduras saturadas & Menor que 10\% do Valor Energético Total \\
\hline Fibras & 7 a 10 gramas \\
\hline Sódio & 720 a 960 miligramas \\
\hline NdPCal & No mínimo 6\% e no máximo $10 \%$ do Valor Energético Total \\
\hline
\end{tabular}

Para avaliar os fatores de riscos das DCNT existentes nos trabalhadores da UAN, realizou-se uma avaliação antropométrica utilizando o Índice de Massa Corporal (IMC), obtido pelo cálculo do peso corporal $(\mathrm{Kg})$ dividido pela estatura ao quadrado $\left(\mathrm{m}^{2}\right)$, e aplicou-se um questionário adaptado do Ministério da Saúde ${ }^{(16)}$.

Para avaliação antropométrica, utilizaram-se os critérios estabelecidos pelo Sistema de Vigilância Alimentar e Nutricional (SISVAN) ${ }^{(17)}$. Para aferição do peso corporal, os funcionários estavam com roupas leves e descalços, e foram posicionados sobre uma balança digital eletrônica, da marca Glife $^{\circledR}$, com precisão de 0,1 quilograma. $\mathrm{Na}$ avaliação da estatura, foi utilizada uma fita métrica de 150 centímetros, com precisão de 0,1 centímetro, a qual foi fixada em uma parede lisa, sem rodapé, a partir da altura de 50 centímetros do chão. Nessa medição, os indivíduos mantiveram os pés unidos, calcanhares encostados na parede, em postura ereta, com olhar fixo no horizonte (ou cabeça ajustada ao plano de Frankfurt), sendo utilizada uma régua sobre a cabeça do avaliado para medir a altura. A classificação do IMC foi realizada de acordo com os pontos de corte utilizados para diagnóstico nutricional de adultos, estabelecidos pela World Health Organization ${ }^{(18)}$.

Posteriormente, aplicou-se um questionário adaptado do Ministério da Saúde ${ }^{(16)}$, com perguntas de múltipla escolha, referentes aos hábitos de vida dos trabalhadores (tabagismo, atividade física, consumo de álcool e patologias), com o objetivo de avaliar os fatores de risco presentes nessa população para desenvolvimento ou existência de morbidades.

Classificou-se o tabagismo em não fumantes, fumantes e ex-fumantes ${ }^{(16)}$. Avaliou-se o sedentarismo pela frequência de atividade física, sendo classificados como ativos quando se exercitavam no mínimo por 30 minutos, cinco vezes por semana, ou mais que 150 minutos semanais. Os que não atendiam a esses critérios foram considerados sedentários ${ }^{(19)}$. O consumo de bebida alcoólica foi avaliado como presente ou ausente, sendo verificada a frequência de ingestão semanal ${ }^{(16)}$.

Os resultados foram analisados por meio de estatística descritiva simples (média e desvio padrão), sendo o trabalho realizado após aprovação no Comitê de Ética em Pesquisa 
do Centro Universitário José Campos Andrade, sob registro $\mathrm{n}^{\circ} .798 .420$.

\section{RESULTADOS}

Após os critérios de inclusão, a amostra do estudo foi composta por 19 funcionários, dos quais quinze desempenhavam funções operacionais e quatro, funções administrativas.

$\mathrm{Na}$ Tabela I, encontra-se o resultado da composição nutricional das refeições oferecidas na UAN. Constatou-se que, na média geral, o VET (1311,7 Kcal), proteínas (19\%), gorduras totais $(32 \%)$, sódio $(1828,6 \mathrm{mg})$, fibras $(21,14$ gramas) e o NdPCal (12\%) das refeições estavam acima dos limites preconizados pelo PAT (Quadro I).

Com relação às variáveis "sexo", "faixa etária" e "IMC" (Tabela II), observou-se que 68\% (n=13) dos funcionários eram homens, a maioria dos participantes da pesquisa $(63 \%, \mathrm{n}=12)$ se encontrava na faixa etária entre 20 e 50 anos, $53 \%(n=10)$ estavam com sobrepeso e $21 \%$ eram obesos ( $\mathrm{n}=4)$.

A avaliação das variáveis "tabagismo", "atividade física" e "consumo de bebida alcoólica" está demonstrada na Tabela III. Observa-se que $42 \%(n=8)$ dos funcionários não tinham hábito de fumar e 37\% (n=7) afirmaram ser exfumantes. Quanto à prática de atividade física, $58 \%(\mathrm{n}=11)$

Tabela I - Avaliação da composição nutricional das refeições servidas no horário do almoço em uma Unidade de Alimentação e Nutrição de uma empresa do ramo de prestação de serviço/automotivo. Curitiba, Paraná, 2014.

\begin{tabular}{lcccccccc}
\hline Dia & VET & $\begin{array}{c}\text { CHO } \\
\mathbf{( \% )}\end{array}$ & $\begin{array}{c}\text { PTN } \\
\mathbf{( \% )}\end{array}$ & $\begin{array}{c}\text { NdPCal } \\
\mathbf{( \% )}\end{array}$ & $\begin{array}{c}\text { GT } \\
\mathbf{( \% )}\end{array}$ & $\begin{array}{c}\text { GS } \\
\mathbf{( \% )}\end{array}$ & $\begin{array}{c}\text { Fibra } \\
\mathbf{( g )}\end{array}$ & $\begin{array}{c}\text { Sódio } \\
(\mathbf{m g})\end{array}$ \\
\hline 1 & 1016,7 & 50 & 16 & 10 & 34 & 14 & 16,5 & 1494,9 \\
2 & 1501,0 & 50 & 14 & 8 & 36 & 10 & 17,3 & 2201,7 \\
3 & 1291,8 & 49 & 20 & 11 & 31 & 8 & 19,1 & 1598,3 \\
4 & 1408,0 & 52 & 19 & 10 & 29 & 6 & 30,4 & 1321,2 \\
5 & 1471,8 & 51 & 12 & 8 & 37 & 8 & 39,4 & 2129,6 \\
6 & 924,5 & 53 & 16 & 9 & 31 & 8 & 31,3 & 2304,1 \\
7 & 911,0 & 60 & 18 & 11 & 22 & 7 & 18,2 & 1751,3 \\
8 & 1401,0 & 40 & 16 & 10 & 44 & 14 & 21,0 & 1990,1 \\
9 & 1494,7 & 35 & 20 & 13 & 45 & 16 & 18,3 & 1734,0 \\
10 & 1685,7 & 57 & 17 & 10 & 25 & 6 & 18,2 & 2363,5 \\
11 & 1009,4 & 56 & 23 & 14 & 21 & 5 & 17,3 & 1776,9 \\
12 & 1209,1 & 39 & 20 & 13 & 41 & 15 & 15,9 & 1716,8 \\
13 & 1230,7 & 39 & 22 & 13 & 39 & 12 & 17,5 & 1870,4 \\
14 & 1444,1 & 49 & 21 & 13 & 30 & 10 & 20,0 & 1929,6 \\
15 & 1595,0 & 43 & 28 & 17 & 29 & 6 & 30,2 & 1453,9 \\
16 & 962,0 & 53 & 24 & 15 & 23 & 9 & 17,2 & 1807,6 \\
17 & 1191,5 & 54 & 24 & 15 & 22 & 4 & 16,9 & 2061,9 \\
18 & 1490,9 & 40 & 19 & 12 & 41 & 16 & 17,2 & 1743,2 \\
19 & 1192,7 & 54 & 18 & 11 & 28 & 6 & 20,7 & 1569,5 \\
20 & 1802,6 & 38 & 24 & 16 & 38 & 8 & 20,0 & 1753,6 \\
\hline Média & 1311,56 & 48,1 & 19,55 & 11,95 & 32,3 & 9,4 & 21,13 & 1828,6 \\
DP & 259,49 & 7,36 & 3,89 & 2,58 & 7,58 & 3,81 & 6,39 & 281,66 \\
\hline
\end{tabular}

VET=Valor Energético Total; $\mathrm{CHO}=$ carboidrato; $\mathrm{PTN}=$ proteína; $\mathrm{NdPCal=percentual} \mathrm{proteico-calórico;} \mathrm{GT}=$ gordura total; GS=gordura saturada; g=grama; \%=percentual; mg=miligrama. $\mathrm{DP}=$ desvio padrão. 
Tabela II - Sexo, faixa etária e índice de massa corporal de funcionários de uma Unidade de Alimentação e Nutrição de uma empresa do ramo de prestação de serviço/automotivo. Curitiba, Paraná, 2014.

\begin{tabular}{lc}
\hline Variáveis & $\mathbf{\%}(\mathbf{n}=\mathbf{1 9})$ \\
\hline Sexo & $68(\mathrm{n}=13)$ \\
$\quad$ Masculino & $32(\mathrm{n}=6)$ \\
Feminino & $11(\mathrm{n}=2)$ \\
Faixa etária & $21(\mathrm{n}=4)$ \\
Menos de 20 anos & $21(\mathrm{n}=4)$ \\
20 a 30 anos & $21(\mathrm{n}=4)$ \\
31 a 40 anos & $26(\mathrm{n}=5)$ \\
41 a 50 anos & \\
51 a 60 anos & $0(\mathrm{n}=0)$ \\
Índice de Massa Corporal & $26(\mathrm{n}=5)$ \\
Menor que $18,5 \mathrm{Kg} / \mathrm{m}^{2}$ & $53(\mathrm{n}=10)$ \\
18,5 a $24,9 \mathrm{~kg} / \mathrm{m}^{2}$ & $16(\mathrm{n}=3)$ \\
25 a $29,9 \mathrm{~kg} / \mathrm{m}^{2}$ & $5(\mathrm{n}=1)$ \\
30 a $34,9 \mathrm{Kg} / \mathrm{m}^{2}$ & $0(\mathrm{n}=0)$ \\
35 a $39,9 \mathrm{~kg} / \mathrm{m}^{2}$ & \\
Maior que $40 \mathrm{~kg} / \mathrm{m}^{2}$ & \\
\hline
\end{tabular}

$\mathrm{n}=$ número da amostra; \%=percentual.

Tabela III - Características dos hábitos de vida de trabalhadores de uma empresa do ramo de prestação de serviço/automotivo. Curitiba, Paraná, 2014.

\begin{tabular}{lc}
\hline Variáveis & $\mathbf{\%}(\mathbf{n})$ \\
\hline Tabagismo (n=19) & $21(\mathrm{n}=4)$ \\
Sim & $42(\mathrm{n}=8)$ \\
Não & $37(\mathrm{n}=7)$ \\
Ex-fumante & $42(\mathrm{n}=8)$ \\
Praticante de atividade física (n=19) & $58(\mathrm{n}=11)$ \\
$\quad$ Sim & $12(\mathrm{n}=1)$ \\
Não & $0(\mathrm{n}=0)$ \\
Frequência da atividade física (n=8) & $88(\mathrm{n}=7)$ \\
Uma vez por semana & $32(\mathrm{n}=6)$ \\
Duas a três vezes por semana & $68(\mathrm{n}=13)$ \\
Mais de três vezes por semana & \\
Consome bebida alcoólica (n=19) & $33(\mathrm{n}=2)$ \\
Sim & $17(\mathrm{n}=1)$ \\
Não & $50(\mathrm{n}=3)$ \\
Frequência de consumo de bebida alcoólica (n=6) & \\
Eventualmente & \\
Uma vez por semana & \\
Mais de duas vezes na semana ou diariamente & \\
\hline
\end{tabular}

$\%=$ percentual; $\mathrm{n}=$ número da amostra.

Tabela IV - Distribuição das patologias relatadas pelos trabalhadores de uma empresa do ramo de prestação de serviço/ automotivo. Curitiba, Paraná, 2014.

\begin{tabular}{lc}
\hline Variáveis & $\mathbf{\%}(\mathbf{n})$ \\
\hline Patologias diagnosticadas $(\mathbf{n}=\mathbf{1 9})$ & $32(\mathrm{n}=6)$ \\
Sim & $68(\mathrm{n}=13)$ \\
Não & \\
Tipos de patologia $(\mathbf{n}=\mathbf{6})$ & $32(\mathrm{n}=6)$ \\
Hipertensão arterial & $11(\mathrm{n}=2)$ \\
\hline Dislipidemia & \\
\hline
\end{tabular}

$\%=$ percentual; $\mathrm{n}=$ número da amostra. 
não a faziam. Com relação ao hábito de beber, $32 \%$ (n=6) consumiam bebidas alcoólicas frequentemente, dos quais $50 \%(\mathrm{n}=3)$ relataram consumir mais de duas vezes por semana ou diariamente.

A distribuição das patologias relatadas pelos funcionários da UAN encontra-se na Tabela IV. Observase que $32 \%(n=6)$ apresentavam algum tipo de patologia, sendo o diagnóstico de hipertensão arterial relatado por todos os participantes.

\section{DISCUSSÃO}

Fornecer uma alimentação adequada para trabalhadores é essencial para a manutenção da saúde e produtividade da empresa ${ }^{(20)}$. Nesse sentido, compete às empresas participantes do PAT assegurar a qualidade da alimentação fornecida aos funcionários, sendo o almoço uma das refeições principais ${ }^{(9)}$, considerada essencial para a promoção da alimentação saudável dos trabalhadores.

Na média geral, o VET das refeições $(1311,7 \mathrm{kcal})$ estava inadequado, pois o PAT estabelece como parâmetro para as refeições principais o valor de 600 a 800 calorias, admitindo-se acréscimo de $20 \%$ do VET de $2.000 \mathrm{Kcal} /$ dia, com total de $1.200 \mathrm{Kcal} /$ dia. O VET das refeições deve ainda corresponder à faixa de $30 \%$ a $40 \%$ do VET diário( ${ }^{(9)}$. $\mathrm{O}$ valor médio do VET encontrado neste estudo foi de $66 \%$, acima do limite estabelecido. Portanto, as refeições oferecidas na UAN estudada não garantem uma alimentação saudável para os trabalhadores, o que pode estar relacionado com a composição das preparações, as quais são compostas por ingredientes com alto teor de gorduras e proteínas, em conjunto com métodos de frituras.

Resultados semelhantes também foram encontrados em outra pesquisa realizada no Brasil, na qual se observou alta densidade calórica nas refeições servidas a trabalhadores, atribuindo esse resultado às ofertas elevadas de gordura, proteína, carboidrato e colesterol ${ }^{(21)}$. O balanço energético positivo associado ao baixo nível de atividade é um dos fatores que auxiliam no aumento da massa corporal dos indivíduos e pode estar relacionado ao excesso de peso presente na população deste estudo ${ }^{(21)}$. Esses fatos reforçam a importância da elaboração de cardápios variados, com aumento na oferta de frutas e hortaliças para contribuir na redução da densidade energética das refeições em UAN.

$\mathrm{Na}$ média geral, o percentual de carboidratos $(48,1 \%)$ apresentou-se abaixo dos parâmetros nutricionais determinados pelo PAT $(60 \%)^{(9)}$. É importante ressaltar que a distribuição do percentual dos nutrientes é baseada na média geral das calorias encontradas nas refeições, o que não sugere necessariamente oferta baixa de carboidratos, e sim inadequação na distribuição do percentual de nutrientes.
Isso ocorre porque os alimentos fontes de carboidratos são diariamente fornecidos por meio da composição do prato base e, frequentemente ( 2 a 3 vezes por semana), ofertados como complemento.

Com relação ao percentual de proteínas e proteicocalórico (NdPCal) (19,55\% e 11,95\%, respectivamente), ambos, na média geral, apresentaram valores acima dos limites estabelecidos pelo PAT, sendo $15 \%$ para proteínas e de $6 \%$ a $10 \%$ para o $\mathrm{NdPCal}^{(9)}$. Esses valores podem estar relacionados à elevada quantidade de carnes fornecidas nas refeições da UAN estudada (duas opções diariamente). O consumo excessivo desse nutriente pode prejudicar a saúde dos indivíduos, pois é considerado a principal fonte de gordura saturada e colesterol, o que pode elevar o risco de aterosclerose $\mathrm{e}^{(22)}$.

O valor médio das gorduras totais $(32,3 \%)$ excedeu o preconizado pela legislação $(25 \%)^{(9)}$. Esse resultado pode ser justificado pela utilização habitual de frituras, cortes de carne ricos em gorduras e outras preparações que apresentam alto teor de lipídios. O consumo excessivo de gordura está associado a mudanças no perfil lipídico sanguíneo, com consequente aumento dos fatores de risco para desenvolvimento de $\mathrm{DCNT}^{(23)}$.

Estudo realizado em 72 empresas na cidade de São Paulo-SP(24), ao avaliar aspectos dietéticos de refeições fornecidas a trabalhadores, também verificou que o valor médio de gordura total foi de $30 \%$. Para mudar essa realidade, é necessária a utilização de métodos de preparação que auxiliem na diminuição de lipídios, tais como assados, cozidos e refogados, bem como escolher ingredientes com menor teor de gordura, como as carnes magras ${ }^{(25)}$.

A oferta de sódio encontrada nas refeições $(1828,6 \mathrm{mg})$ estava acima dos limites estabelecidos pelo PAT (720-960 $\mathrm{mg}$ ) para as principais refeições (almoço, jantar e ceia) ${ }^{(9)}$, o que pode estar relacionado com a utilização de temperos prontos à base de sódio (caldo de carne, extrato de tomate e shoyu), embutidos (linguiça e bacon) e sal de cozimento. Para diminuir o consumo de sódio nas refeições servidas em UANs, recomenda-se a utilização de Fichas Técnicas de Preparação, pois auxiliam no controle e substituição de temperos industrializados (caldos concentrados) e sal de adição por temperos naturais (ervas secas, cebola, alho, especiarias) marinados e caldos naturais que realçam o sabor dos alimentos ${ }^{(26)}$.

Vale ressaltar, ainda, que a quantidade de sódio encontrada nas refeições avaliadas neste estudo está próxima ao valor recomendado pela OMS para o dia inteiro $(2000 \mathrm{mg})^{(27)}$, demonstrando que apenas uma refeição está suprindo quase toda a demanda diária de sódio. Isso deve ser modificado, pois o consumo excessivo desse mineral está associado ao aumento da prevalência da hipertensão arterial 
sanguínea, e considera-se um importante fator de risco para o aumento da mortalidade por doenças cardiovasculares ${ }^{(23)}$.

A quantidade média de fibra encontrada nas refeições $(21,13 \mathrm{~g})$ foi alta, na ordem de duas a três vezes maior que a recomendação pelo $\mathrm{PAT}^{(9)}$. Esse resultado é insatisfatório, pois o consumo de fibras, apesar de ser importante no controle da glicemia, metabolismo de lipídios e contribuir para o bom funcionamento do trânsito intestinal, saciedade e controle do peso ${ }^{(28)}$, quando em excesso pode acarretar alguns problemas, tais como desidratação, desconforto intestinal e limitação da absorção de ferro, cálcio e outros nutrientes, podendo resultar em deficiências nutricionais ${ }^{(29)}$.

O percentual de gordura saturada nas refeições $(9,4 \%)$ estava dentro dos parâmetros determinado pelo PAT $(<10 \%)$ (9), o que é satisfatório, pois o consumo de gorduras saturadas acima do limite permitido está relacionado a alterações no perfil lipídico sanguíneo e progressão de doenças, como a diabetes mellitus tipo 2 e obesidade ${ }^{(22)}$.

Constatou-se que mais da metade dos funcionários estavam com sobrepeso e obesidade. Esses resultados são inesperados para trabalhadores que desempenham atividades intensas, como os avaliados neste estudo. O cenário da prevalência de sobrepeso e obesidade também foi encontrado em outras pesquisas com trabalhadores no Brasil $^{(8,13)}$. Esses resultados podem estar relacionados com escolhas alimentares inadequadas, tanto na empresa como fora dela, e demonstra que ações mais efetivas devem ser realizadas com o intuito de diminuir o impacto do atual cenário do país em relação a essas doenças.

A maioria dos funcionários não era fumante ou afirmou ser ex-fumante. O que é satisfatório, pois o tabagismo é considerado um importante problema de saúde pública, devido à alta mortalidade das doenças relacionadas ao seu uso. Segundo levantamento realizado por inquérito telefônico no país, houve uma queda de fumantes do ano de 2006 a 2013 , quando a taxa de $15,6 \%$ passou para $11,3 \%{ }^{(16)}$, o que corrobora com os achados deste estudo e demonstra que o consumo de tabaco vem diminuindo no país.

A ausência da prática de atividade física foi relatada por mais da metade dos trabalhadores. O que é inadequado, pois sua prática é recomendada e pode auxiliar no controle da hipertensão arterial, além da manutenção do peso corporal e prevenção da diabetes mellitus tipo $2^{(25)}$.

O consumo de bebidas alcoólicas foi avaliado como frequente por alguns trabalhadores, que relataram consumir mais de duas vezes por semana ou diariamente. Esses resultados são semelhantes ao encontrado em FortalezaCE, com 156 comerciários, dos quais $36,54 \%$ relataram consumir bebida alcoólica frequentemente ${ }^{(30)}$.

Neste estudo, a hipertensão arterial foi relatada por todos os funcionários que apresentavam alguma patologia, o que pode ter relação direta com a elevada quantidade de sódio encontrado nas refeições e demonstra seu desacordo em relação aos objetivos do PAT e sua implementação nas empresas.

O PAT é considerado um programa essencial para políticas de alimentação e nutrição voltadas para a população trabalhadora. Aplicado corretamente, pode contribuir efetivamente para a transformação do atual panorama de aumento da prevalência de DCNT e obesidade no país. Nesse sentido, ações devem ser realizadas para proporcionar aumento no conhecimento dos trabalhadores beneficiados, assim como dos gestores administrativos e técnicos, para sua efetiva operacionalização e promoção da alimentação saudável aos trabalhadores.

\section{CONCLUSÃO}

As refeições servidas na Unidade de Alimentação e Nutrição avaliada estavam inadequadas aos parâmetros estabelecidos para a alimentação do trabalhador, pois apresentavam excesso de calorias, inadequações de alguns nutrientes (carboidratos, proteínas, gorduras totais, gorduras saturadas, fibras e sódio) e do NdPCal. Isso pode acarretar prejuízo à saúde dos beneficiários quando associado aos principais fatores de riscos encontrados, como sobrepeso, sedentarismo e prevalência de hipertensão arterial, além do não cumprimento à legislação trabalhista brasileira.

\section{REFERÊNCIAS}

1. Ministério da Saúde (BR). Diretrizes e recomendações para o cuidado integral de doenças crônicas nãotransmissíveis: promoção da saúde, vigilância, prevenção e assistência. $1^{\text {a }}$ ed. Brasília: Ministério da Saúde; 2008 [acesso em 2015 Mar 15]. Disponível em: http://bvsms.saude.gov.br/bvs/publicacoes/diretrizes_ recomendacoes_cuidado_doencas_cronicas.pdf

2. Ministério da Saúde (BR), Secretaria de Vigilância em Saúde, Departamento de Análise de Situação de Saúde. Plano de ações estratégicas para o enfrentamento das doenças crônicas não transmissíveis (DCNT) no Brasil 2011-2022. Brasília: Ministério da Saúde; 2011 [acesso em 2015 Mar 10]. Disponível em: http://bvsms. saude.gov.br/bvs/publicacoes/plano_acoes_enfrent_ dent_2011.pdf

3. Organização Mundial da Saúde. Global status report on non communicable diseases: 2010. Genebra: OMS; 2011. [acesso em 2015 Fev 21]. Disponível em: http:// www.who.int/nmh/publications/ncd_repornt2010/en/

4. Ministério da Saúde (BR). Diretrizes para o cuidado das pessoas com doenças crônicas nas redes de atenção 
à saúde e nas linhas de cuidado prioritárias. Brasília: Ministério da Saúde, 2013 [acesso em 2015 Fev 21]. Disponível em: http://bvsms.saude.gov.br/bvs/ publicacoes/diretrizes\%20_cuidado_pessoas $\% 20$ doencas_cronicas.pdf

5. Ministério da Saúde (BR). Plano de Ações Estratégicas para o Enfrentamento das Doenças Crônicas Não Transmissíveis (DCNT) no Brasil 2011-2022. Brasília: Ministério da Saúde; 2011. (Série Eletrônica) [acesso em 2015 Mar 12]. Disponível em: http://189.28.128.100/ dab/docs/geral/plano_dent.pdf

6. Ministério do Trabalho e Emprego (BR). Lei nº. 6.321, de 14 de abril de 1976. Dispõe sobre a dedução, do lucro tributável para fins de imposto sobre a renda das pessoas jurídicas, do dobro das despesas realizadas em programas de alimentação do trabalhador. Diário Oficial da República Federativa do Brasil, Brasília, abr. 1976. [acesso em 2015 Mar 12]. Disponível em: http:/ www.planalto.gov.br/ccivil_03/leis/L6321.htm.

7. Ministério do Trabalho e Emprego (BR). Decreto $\mathrm{n}^{\circ}$. 05, de 14 de janeiro de 1991. Regulamenta a Lei ${ }^{\circ}$ 6.321, de 14 de abril de 1976, que trata do Programa de Alimentação do Trabalhador, revoga o Decreto $\mathrm{n}^{\circ} 78.676$, de 8 de novembro de 1976, e dá outras providências. Diário Oficial da República Federativa do Brasil, Brasília, jan. 1991. [acesso em 2015 Mar 12]. Disponível em: http://www.planalto.gov.br/ccivil_03/ decreto/1990-1994/D0005.htm

8. Lanci KNS, Matsumoto KL. Avaliação nutricional e análise do cardápio oferecido para funcionários beneficiados pelo programa de alimentação do trabalhador em uma indústria de paranavaí, Paraná. SaBios (Campo Mourão. Online). 2013;8(3):3-12.

9. Ministério do Trabalho e Emprego (BR). Portaria Interministerial $\mathrm{n}^{\circ} .66$, de 25 de agosto de 2006. Altera os parâmetros nutricionais do Programa de Alimentação do Trabalhador (PAT). Diário Oficial da República Federativa do Brasil, Brasília, ago. 2006. [acesso em 2015 Mar 12]. Disponível em: http://189.28.128.100/ nutricao/docs/legislacao/portaria66_25_08_06.pdf

10. Ministério do Trabalho e Emprego (BR). Portaria Interministerial n. 193, de 5 de dezembro de 2006. Altera os parâmetros nutricionais do Programa de Alimentação do Trabalhador (PAT). Diário Oficial da República Federativa do Brasil, Brasília, dez. 2006. [acesso em 2015 Mar 12]. Disponível em: http:// www.carvaomineral.com.br/abcm/meioambiente/ legislacoes/bd_carboniferas/seguranca_e_medicina_ do_trabalho/portaria_sit_dsst_193-2006.pdf
11. Instituto Brasileiro de Geografia e Estatística - IBGE. Pesquisa de orçamentos familiares (POF): tabelas de composição nutricional dos alimentos consumidos no Brasil 2008-2009. Rio de Janeiro: IBGE; 2011.

12. Camargo EB, Botelho RA. Técnica dietética: seleção e preparo dos alimentos. São Paulo: Atheneu; 2010.

13. Mesquita FLFM, Mesquita AU. Perfil antropométrico, socioeconômico e de saúde de funcionários assistidos pelo programa de alimentação do trabalhador. Rev Bras Promoç Saúde. 2013;26(2):192-200.

14. Universidade Estadual de Campinas. Tabela Brasileira de composição de alimentos (TACO). $4^{\mathrm{a}}$ ed. São Paulo: UNICAMP; 2011 [acesso em 2015 Mar 10]. Disponível em: http://www.unicamp.br/nepa/taco/contar/taco_4 edicao_ampliada_e_revisada.pdf?arquivo $=$ taco_ 4 versao_ampliada_e_revisada.pdf

15. Abreu ES, Spinelli MGN, Pinto AMS. Gestão de unidades de alimentação e nutrição: um modo de fazer. $4^{\mathrm{a}}$ ed. São Paulo: Editora Metha; 2011.

16. Ministério da Saúde (BR). Vigitel Brasil 2013: vigilância de fatores de risco e proteção para doenças crônicas por inquérito telefônico. Brasília: Ministério da Saúde; 2014 [acesso em 2015 Abr 26]. Disponível em: $<$ http://biavati. files.wordpress.com/2014/05/vigitel-2013.pdf

17. Ministério da Saúde (BR). Orientações para a coleta e análise de dados antropométricos em serviços de saúde: Norma Técnica do Sistema de Vigilância Alimentar e Nutricional (SISVAN). Brasília: Ministério da Saúde; 2011 [acesso em 2015 Abr 12]. Disponível em: http://189.28.128.100/dab/docs/portaldab/publicacoes/ orientacoes_coleta_analise_dados_antropometricos. pdf

18. World Health Organization - WHO. Physical status: the use and the interpretation of an anthropometry: report of a WHO expert comitee. Genebra: WHO; 1995.

19. Organización Mundial de La Salud. Recomendaciones mundiales sobre actividad física para la salud. Ginebra: OMS; 2010 [acesso em 2015 Abr 15]. Disponível em: http://whqlibdoc.who.int/publications /2010/9789243599977_spa.pdf

20. Henriques P, Andrade AS, Tavares MF, Sampaio RM. Editais públicos para o fornecimento de pequenas refeições e o Programa de Alimentação do Trabalhador: um estudo de caso. Nutrire Rev Soc Bras Aliment Nutr. 2014;39(1):56-67.

21. Associação Brasileira para o Estudo da Obesidade e da Síndrome Metabólica. Diretrizes brasileiras de 
obesidade 2009/2010. $3^{\text {a }}$ ed. Itapevi: 2009 [acesso em 2015 Abr 20]. Disponível em: http://www.abeso.org. br/pdf/diretrizes_brasileiras_obesidade_2009_2010_1. pdf

22. Sociedade Brasileira de Cardiologia; Sociedade Brasileira de Hipertensão; Sociedade Brasileira de Nefrologia. VI Diretrizes brasileiras de hipertensão. Arq Bras Cardiol. 2010;95(Supl 1):1-51.

23. Martins DMS, Broilo MC, Zani VT. Óleos e gorduras: utilização no processo produtivo de restaurantes comerciais de Porto Alegre, Brasil. Nutrire Rev Soc Bras Aliment Nutr. 2014;39(1):25-39.

24. Geraldo APG, Bandoni DH, Jaime PC. Aspectos dietéticos das refeições oferecidas por empresas participantes do Programa de Alimentação do Trabalhador na Cidade de São Paulo, Brasil. Rev Panam Salud Pública. 2008;23(1):19-25.

25. Salas CKTS, Spinelli MGN, Kawashima LM, Ueda AM. Teores de sódio e lipídios em refeições almoço consumidas por trabalhadores de uma empresa do município de Suzano, SP. Rev Nutr. 2009;22(3): 331-39.

26. Agência Nacional de Vigilância Sanitária (BR). Guia de boas práticas nutricionais: restaurantes coletivos. Brasília: ANVISA; 2014 [acesso em 2015 Abr 25]. Disponível em: http://portal.anvisa.gov.br/wps/wcm/ connect/a9cfa50046274126b652bfec1b28f937/ Guia + de+Boas + Pr\%C3\%A1ticas + Nutricionais + para + Restaurantes + Coletivos. pdf?MOD=AJPERES

27. World Health Organization - WHO. Guideline: Sodium intake for adults and children. Geneva: WHO; 2012. [acesso em 2015 Mar 12]. Disponível em: http://www. who.int/nutrition/publications/guidelines/sodium intake_printversion.pdf

28. Sociedade Brasileira de Diabetes. Diretrizes da sociedade brasileira de Diabetes 2009. $3^{\mathrm{a}}$ ed. Itapevi: Araújo Silva Farmacêutica; 2009.

29. Florindo AA, Romero A, Peres SV, Silva MV, Slater B. Desenvolvimento e validação de um questionário de avaliação da atividade física para adolescentes. Rev Saúde Pública. 2006;40(5):802-9.

30. Alves JWS, Soares NT, Leão TCS, Diniz NA, Penha EDS, Monteiro RS. Estado nutricional, estilo de vida, perfil socioeconômico e consumo alimentar de comerciários. Rev Bras Promoç Saúde. 2012;25(2): 40-50.

\section{Endereço para correspondência:}

Priscila de Lima Batista

Rua: Alameda Dr. Muricy, 706

Bairro: Centro

CEP: 80010-120 - Curitiba - PR - Brasil

E-mail: priscilal.b@hotmail.com 